\title{
Opportunities and Challenges for Data Physicalization
}

\author{
Yvonne Jansen ${ }^{1}$, Pierre Dragicevic ${ }^{2}$, Petra Isenberg ${ }^{2}$, Jason Alexander ${ }^{3}$, \\ Abhijit Karnik $^{3}$, Johan Kildal ${ }^{4}$, Sriram Subramanian ${ }^{5}$, Kasper Hornbæk ${ }^{1}$ \\ ${ }^{1}$ University of Copenhagen, ${ }^{2}$ Inria, ${ }^{3}$ Lancaster University, ${ }^{4}$ Nokia, ${ }^{5}$ University of Bristol \\ jansen.yv@gmail.com, \{pierre.dragicevic, petra.isenberg\}@inria.fr, \{j.alexander, \\ a.karnik\}@lancaster.ac.uk,johan.kildal@nokia.com, sriram@cs.bris.ac.uk,kash@di.ku.dk
}
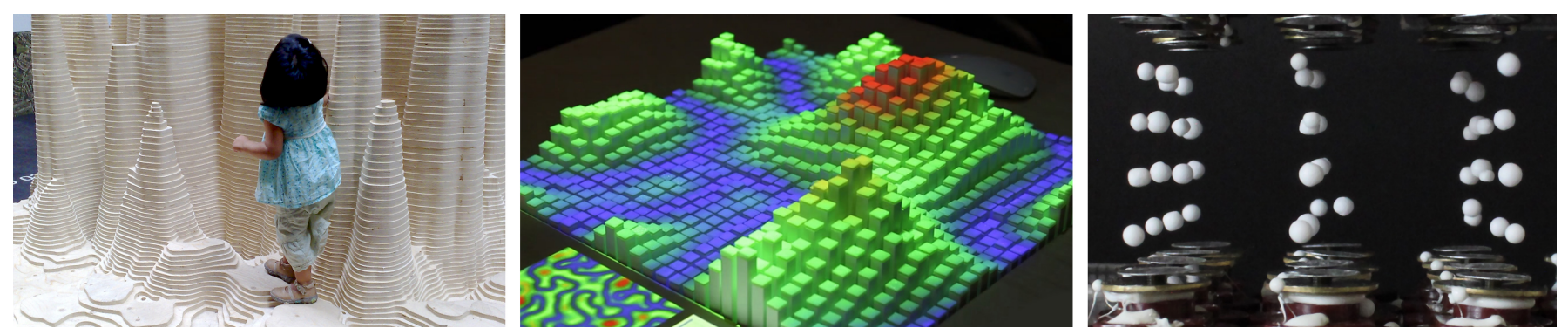

Figure 1: Examples of data physicalizations: (left) population density map of Mexico City co-created by Richard Burdett and exhibited at the Tate Modern (photo by Stefan Geens), (center) similar data shown on an actuated display from the MIT Media Lab [70], and (right) spherical particles suspended by acoustic levitation [61]. All images are copyright to their respective owners.

\section{ABSTRACT}

Physical representations of data have existed for thousands of years. Yet it is now that advances in digital fabrication, actuated tangible interfaces, and shape-changing displays are spurring an emerging area of research that we call Data Physicalization. It aims to help people explore, understand, and communicate data using computer-supported physical data representations. We call these representations physicalizations, analogously to visualizations - their purely visual counterpart. In this article, we go beyond the focused research questions addressed so far by delineating the research area, synthesizing its open challenges, and laying out a research agenda.

\section{Author Keywords}

Data physicalization; visualization; tangible user interfaces; shape-changing interfaces; physical visualization

\section{ACM Classification Keywords}

H.5.m. Information Interfaces and Presentation (e.g., HCI): Miscellaneous

\section{INTRODUCTION}

More than 7000 years ago, the Sumerians used clay tokens to represent quantitative data well before inventing written language [59]. The Incas never even developed a writing system but relied on Quipus - complex assemblies of knotted

This is the author's version of the work. It is posted here for your personal use. Not for redistribution. The definitive version of Record was published in CHI ' 15 which can be found at:

http://dx.doi.org/10.1145/2702123.2702180 ropes - for data storage and retrieval [3]. Scientists in the $19^{\text {th }}$ century used physical representations as essential teaching and research aids [15] for organic chemistry and thermodynamics. Today, designers are crafting data sculptures to convey meaning and emotion in data [79]. All such physical representations help people think about, explore, and share data.

Similarly, for centuries people have been developing visualizations for flat surfaces, such as maps or genealogies printed on papyrus, paper, tapestries, and now computer screens. Yet, recent and anticipated technological advances in digital fabrication, tangible user interfaces [63], shape displays [54], and programmable matter [30] are radically changing the fidelity and flexibility with which data can be made physical (see Figure 1) while decreasing the cost of doing so. We see an unprecedented opportunity to create new forms of physical representations - they will be self-reconfigurable, freely manipulable, and will eventually support data analysis tasks as complex as performed on today's desktop computers.

This article identifies Data Physicalization as an emerging research area that uses physical data representations to help people explore and communicate data. So far work in this area has been scattered and has focused on specific questions, thus an overview is needed to establish this area and to advance its research. We illustrate the current and future potential of physicalizations through usage scenarios and discuss their possible benefits. We clarify how this area relates to other domains such as Visualization [10] and Tangible User Interfaces [31] by illustrating its focus on data analysis (versus other forms of interface usage) in a physical form (versus visual presentation only). We then synthesize a research agenda for Data Physicalization detailing challenges such as how to encode data physically, how to support interaction, what enabling technologies are needed, and how to evaluate physicalizations. 


\section{WHAT ARE DATA PHYSICALIZATIONS?}

We propose that:

A data physicalization (or simply physicalization) is a physical artifact whose geometry or material properties encode data.

For instance, the physicalization in Figure 1-left is a wooden three-dimensional model of Mexico City where height encodes population density. The photo in the middle shows a physicalization of similar data, but dynamic. Here, the physicalization is used as a detail view to explore a larger information space displayed on an adjacent large screen [70]. While the photo on the right is a technology demonstration, it shows the potential to use free-floating particles to encode data [61].

The above definition should be taken as a working definition. A similar definition has been given for physical visualizations [34], and the terms can be used interchangeably. We prefer physicalization as it aligns with neologisms such as sonification and haptification [56] without favoring one specific sense. By extension, we also refer to physicalization as the process of producing physicalizations (to physicalize is to give data physical shape), and as a research area. These three meanings are consistent with the way visualization is currently informally employed by the Visualization community. However, the lack of an explicit distinction can easily generate confusions that we wish to avoid here. To reinforce this we use capitalization when referring to research areas.

\section{Other Examples of Physicalizations}

The idea of turning data into physical artifacts is not new, with many physicalizations already in everyday use [15]. Today's physicalizations are mostly static, but can offer potential perceptual, cognitive, and communicative value that neither paper nor computer displays may be able to offer.

An example of current use of physicalizations is shown in Figure 2-left, where Kevin Quinn, a chief engineer at General Motors, uses a custom Lego-based board to oversee and update problems and progress in production lines (tinyurl.com/mwilson2012). To him, two important benefits of this physicalization are the constant availability of information and the motivational factor for his engineers who like to see the progress of their work publicly reflected on the board.

Medical doctor and public speaker Hans Rosling (Figure 2right) uses physical representations to emphasize his messages during his presentations (tinyurl.com/roslingBox). He uses physicalizations for their communicative and perhaps persuasive power. In the shown example, he discusses the divide between the world's rich (blue box) and poor (green boxes) and illustrates possible effects of policy changes by moving these boxes between piles. His physicalizations are useful and effective in communicating information which individuals may find difficult to comprehend due to unfamiliar magnitudes and scales. It can also carry a shock value.

\section{Future Usage Scenarios For Physicalizations}

To further illustrate the potential of data physicalizations, we provide three usage scenarios which are fictional and to a large extent ignore current technological limitations.
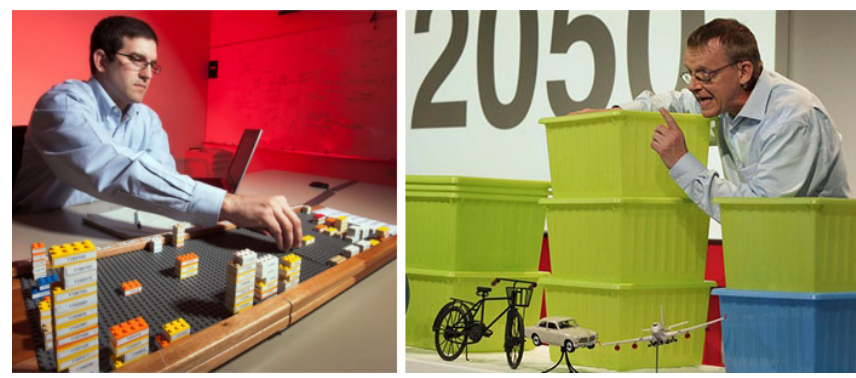

Figure 2: Manually-updated physicalizations used by an automotive engineer (left, image (c) General Motors) and by Hans Rosling during a public talk (right, image (c) TED).

\section{Science Museum}

A visitor to a museum walks into a room which describes the earth's changes in climate. She picks up stones that physicalize the change of temperatures on Earth backwards in time. She can get a good sense of the differences between ice ages and hotter periods - but when she gets to the volcanic beginnings and the stones quickly heat up, she drops them. At the hurricane diorama, miniature hurricanes of the past 50 years move over a map of North America covered in dense fog, with only the eyes of the storms allowing a peak inside. The visitor places her hands over two of the eyes, and the differences in pressure give her the sensation of the intensity of the hurricanes at their peak. She reaches into the eye of the stronger hurricane and feels a strong drag on her hand when it crosses the eyewall. She can easily judge how devastating it was.

\section{Explaining Complex Systems}

A government analyst developed an improved multi-variate model that predicts the economic position of her country. During a meeting, she physicalizes past and predicted time-series data for indicators such as GDP, income tax rates, and unemployment, into line charts (physicalized as wires). Uncertainties in the system are illustrated by wires expanding into cones that cover the ranges of plausible values. To illustrate the requirements for ensuring a 2\% growth in GDP, the analyst deforms and compresses the GDP cone and shows the effect on tax revenue. Once she has reached a realistic growth objective, the dynamic physicalization is frozen and static 3D printed models are created for the policy makers in the audience.

\section{Neurosurgical Planning}

A neurosurgeon scans the brain of a patient who suffers from an asymptomatic tumor. He physicalizes the data into a visually and haptically realistic brain model made of programmable matter (i.e., microscopic particles that can dynamically re-arrange themselves and change their properties [30]). With a caress, he makes the brain semi-translucent to reveal the tumor's localization. He then takes a scalpel and cuts the brain into slices. He lays the slices side-by-side to identify and mark the tumor's tendrils. He then stacks the slices again and they re-assemble back into a monolithic object. With a poking gesture, he then turns the brain into a 3D connectivity graph to reveal functional correlations between brain regions. He pulls dense clusters of links apart to see them more clearly. After this sequence of explorations he gains the insight that the tumor may soon affect important perceptual and cognitive brain functions and recommends immediate scheduling of surgery. 


\section{THE BENEFITS OF DATA PHYSICALIZATION}

We see many possible benefits of physicalization on the perceptual, cognitive, and societal levels. We discuss some of them here. Currently, however, the empirical literature offers scant evidence about the merits and possible limitations of data physicalizations. Consequently, this discussion draws upon the insights from other fields. We later identify the lack of empirical studies as a major element of the research agenda for Data Physicalization. For now, we note that these potential benefits need to be traded off against the costs of data physicalizations, in particular the cost of creating them.

\section{Leveraging our Perceptual Exploration Skills}

Humans have evolved a highly complex sensorimotor system that allows them to efficiently extract information from the physical world [9]. We explain why physicalizations can tap into these skills more deeply than classical computer setups.

Active Perception: A major benefit of physicalizations is that they better exploit our active perception skills. Perception has evolved as an active process, that is, it strongly relies on motor control [20]. For example, a physical object like a hand-sized physicalization can be visually inspected by turning it around, by moving it closer, or by taking it apart. A large-scale physicalization can be explored by walking around. In contrast, on-screen visualizations need to explicitly support active perception by coupling input with output devices, that is, by being "interactive", possibly introducing usability issues. For example, visual exploration of large information spaces typically requires navigation tools such as pan-and-zoom. Although powerful, these tools are often inconsistent across applications and constantly need to be rediscovered and learned.

Depth Perception: Another benefit of physicalizations is their better leverage of our spatial perception skills. Since physical objects give rich cues of shape and volume, 3D data can be perceived with less effort and more accuracy on physicalizations than on computer displays - even stereoscopic ones [34].

Non-visual Senses: While vision is a dominant sense, it is not the only way by which we explore the world. As our earlier scenarios illustrated, touching a physical object can reveal a whole set of additional information such as texture, stiffness, temperature, and weight [27] - it becomes a haptic display of information [38]. Hearing also provides information on object properties [11]. Even smell or taste can can signal warning or invitation. All senses can participate in information gathering and they each have unique characteristics that can be leveraged by physicalizations. Touch, for example, requires close contact with the object, meaning that materials can be manipulated to influence our emotional states [27]. Physicalizations can take advantage of these additional sensory channels to convey a larger range of meanings than a simple visual display.

Intermodal Perception: The idea of conveying data to several senses has been around (e.g., $[26,42,56])$ but has typically followed a multimodal approach, where multiple output devices address the senses separately. In contrast, data physicalization follows an intermodal approach [64] that guarantees cohesive and realistic multisensory experiences. When inspecting physical objects, perceptual senses are tightly coupled and work hand-in-hand. For example, stroking a surface produces sounds that nuance tactile information on texture [9, Chap 7]. A rich repertoire of gestures is available for gathering both visual and non-visual information. Lateral hand motions can reveal an object's texture, holding it unsupported can reveal its weight, while following contours can reveal the details of its shape [38]. Informative sounds can also be produced by knocking an object or by scratching its surface $[4,11]$. These are all "built-in interaction techniques" and none of them need to be explicitly implemented and documented. Such interactions are hard to emulate with output devices (e.g., [35]).

\section{Making Data Accessible}

Since physicalizations can address non-visual senses, they can make data analysis more accessible to visually-impaired people. Traditional approaches use raised paper to convey maps and charts $[12,16]$, or cork boards with pins and rubber bands to teach mathematical functions [41]. Novel interactive systems are trying to improve these techniques [41,42] but often follow a multimodal approach and thus do not fully leverage our perceptual exploration skills. With physicalizations, visually-impaired data analysts may be able to explore data through the geometry and material properties of data artifacts in more ways than possible with currently established techniques. For instance, they could explore physicalizations with both hands to gain an overview, possibly using tactile pre-attentive cues [10] to identify interesting features that they could then explore in detail. At the same time, such physicalizations could be designed to remain compatible with visual exploration and thereby foster collaboration between sighted and unsighted analysts.

\section{Cognitive Benefits}

We expect physicalizations to benefit cognition and learning. Physical representations have been widely used in classrooms, for example, to teach basic mathematics [19] and chemistry [77]. A large body of evidence from educational psychology suggests that manipulable physical representations facilitate understanding and learning [48]. Part of this research is motivated by the embodied cognition thesis, according to which cognition is supported by the body and the physical world [7]. Similar arguments have been made for the benefits of tangible user interfaces $[48,63]$. Although the artifacts used in classrooms and educational psychology studies are typically not physicalizations (i.e., they do not convey data), we assume that some of their cognitive and educational benefits will transfer to physicalizations. Given the key role of physical manipulation in learning, we believe this to be especially true for rearrangeable and interactive physicalizations [33].

\section{Bringing Data into the Real World}

Apart from their perceptual and cognitive benefits, physicalizations allow individuals and groups to relate to data in new ways. The Tangible User Interface community has already pointed out that embedding physical artifacts in the space around us offers possibilities beyond those of virtual objects [28]. In comparison to screen-based visualizations, physicalization can similarly offer to bring data to many more places and aspects of our social lives. In particular, since physicalizations can be anywhere and are always "on", they can be used as 
ambient data displays and support casual visualization [53]. Furthermore, data is increasingly present in museums and art galleries in the form of data sculptures [15], that is, physicalizations created by designers and artists whose purpose is to communicate meaning and elicit reflection on data [43]. Meanwhile, more and more makers and fabrication enthusiasts physicalize data for sharing or for personal purposes [68]. Even fashion is being impacted, as people are already wearing personal data in the form of jewelery (e.g., www.meshu.io).

\section{Engaging People}

An emerging challenge in information visualization - particularly in the area of data journalism - is to engage large audiences with data [14]. There is anecdotal evidence that physicalizations may aid individuals in engaging with and communicating information to others more effectively than with digital representations. Our earlier example showed Hans Rosling using physicalizations to communicate world statistics (see Figure 2-right). The growing popularity of data physicalizations in many societal domains indicates a strong potential for fostering public engagement. This popularity suggests that data physicalizations pique interest, and this interest could in turn be leveraged to have people spend more time and effort exploring and understanding important and complex data.

\section{Summary}

We have put forward several arguments suggesting that physicalizations can be beneficial. We do not expect them to be beneficial in all situations, but the number of favorable situations will undoubtedly increase as technology advances. The increasing popularity of physicalizations provides another argument for studying them, irrespective of their practical merits and drawbacks. Flat surfaces - such as paper and later computer screens - have long been the primary media for visualization, but this is rapidly changing. While data physicalization is becoming part of our society, scientific research is lagging behind. The TUI community is only starting to consider data exploration scenarios [18], while the Visualization community only recently started to explore physical designs [75] and carry out studies on physicalizations $[34,66]$.

\section{DATA PHYSICALIZATION AS A RESEARCH AREA}

So far we covered data physicalizations as artifacts. We discussed why such artifacts can be beneficial, and hinted at how current and future technology will allow us to create more powerful interactive data physicalizations.

We now discuss Data Physicalization as a research area. We show that it is not simply a subset of existing fields, but a new research area that opens up new research questions and challenges beyond what existing fields are concerned with.

\section{We propose to think of Data Physicalization as:}

a research area that examines how computer-supported, physical representations of data (i.e., physicalizations), can support cognition, communication, learning, problem solving, and decision making.

Data Physicalization relates to two broad topics: (i) the support of data analysis through visualization and (ii) the support of human-computer interaction through physical objects.

\section{Data Physicalization and Visualization}

Data Physicalization is related to the fields of Information Visualization (InfoVis) and Scientific Visualization (SciVis). Since the two fields are most often separated by the type of data they address [44], we use Visualization as an umbrella term for "the use of computer-supported, interactive, visual representations of data to amplify cognition" [10]. This definition includes four aspects closely related to Data Physicalization: a focus on external representations, computing, interaction, and the ultimate goal of enhancing humans' cognitive abilities to deal with data. One key difference is that Data Physicalization does not explicitly focus on the visual channel but instead attempts to tap into perceptual exploration skills more broadly.

Another important difference is that Data Physicalization excludes systems that convey data solely through flat visual displays, although it does include hybrid setups such as deformable visual displays $[1,17,58,65]$, provided the geometry or material properties of the display surface encode data. Consistent with our definition of data physicalizations, the focus is on the physicality of data representation, not on the physicality of interaction. Thus most setups involving multi-touch and large display surfaces are not the focus of Data Physicalization, despite the "physicality" of direct touch interaction and locomotion in space. However, the field is clearly concerned with how touch - both as input and sensory modality - and locomotion can support data analysis with physicalizations.

While Data Physicalization is concerned with physicalizations whose construction or actuation is computer-supported, interaction with physicalizations can also take place in the physical world without computer assistance [34]. Furthermore, handmade physicalizations of which many historical examples exist $[3,15,59]$ may not involve computers at all, but are relevant to Data Physicalization when they can inform and inspire the design of modern, computer-supported physicalizations.

\section{Data Physicalization and TUI}

The area of Tangible User Interfaces (TUI) studies interactive systems that "give physical form to digital information, employing physical artifacts both as representations and controls for computational media" [71]. While this view clearly emphasizes the physical representation of information, the physical representations themselves are generally not meant for data exploration and analysis tasks. Several TUI systems have been developed for such tasks, but they typically use physical objects as controls, while the data itself is visualized on a screen [72] or top-projected $[49,73]$. Although controls can encode information through their physical arrangement, this information entirely originates from the user and is not meant to be explored to gain insights as in Figure 2-left. Thus it does not constitute data in the Visualization sense [10].

Overall, there is large overlap between TUI and Data Physicalization, though they differ in focus. While both necessarily involve input and output and share many concerns, the former mostly focuses on information input and manipulation tasks (with output being used to assist in the task) while the latter mostly focuses on information output and exploration tasks (with input being used to assist in the task). The area of TUI has been remarkably productive in addressing technological 
and theoretical challenges, and its work will be important both to implement data physicalizations and inform their design. Conversely, simple data exploration scenarios have started to be used to illustrate actuated TUIs [70], suggesting that the idea of data physicalization is making its way into the community and may well become a major drive for TUI research.

\section{Examples of Data Physicalization Research}

Several research papers have already started to address questions raised by Data Physicalization. Early on, Vande Moere and colleagues pointed out the potential of data sculptures as a medium and the need to study them more deeply $[43,75,79]$. Later, several papers were published in Visualization and HCI venues that investigate empirical [34], design [67,69], and conceptual $[32,33,75,79]$ questions regarding physicalizations, as well as specific application domains [68]. However, the physicalizations investigated are static and none of this work involves novel input and output technologies. Conversely, as far as we know, none of the work on new user interface technologies explicitly addresses Data Physicalization questions.

\section{ENABLING TECHNOLOGIES}

The area of Data Physicalization both depends heavily on available technologies, and can contribute to shaping the emergence of future technologies. Research has already demonstrated the process for composing and creating one-off static physicalizations using fabrication technologies $[68,69]$. Dynamic physicalizations, on the other hand, require computer-driven control of physical geometry or of material properties.

A range of technologies for controlling physical geometry have been explored in the area of shape-changing interfaces [54,58], many of which could be used to implement dynamic data physicalizations. Several systems offer discretized shape control through arrays of motorized bars $[18,52]$, winch-actuated wires [15], or tiltable tiles [1]. Complex dynamic geometries can be also created by assembling actuated modules $[30,60]$. Alternatively, continuous shape deformation can be achieved through, e.g., pneumatic or hydraulic actuation $[17,78]$, or shape-memory alloys [58]. Microrobotics can also be used to position multiple physical objects on a 2D plane $[21,45,57]$, while free 3D positioning through levitation is currently being researched $[39,61]$. Physical geometry encoding can be complemented with color encoding through the use of deformable visual displays $[47,65]$, sets of actuated visual displays [1], or projection mapping [5]. Finally, technologies also exist for dynamically controlling material properties such as stiffness [17,35,54], weight [46], or transparency [17], all of which can be used as additional dimensions to encode data.

While many of the above systems are bulky and only allow limited control over geometry and material properties, they lay the foundation for the promise of programmable matter [30].

\section{RESEARCH AGENDA}

Data Physicalization brings with it many research questions and challenges regarding the design, implementation, and evaluation of physicalizations. We split up open research questions into four main themes: (i) how to convey data effectively, (ii) how to employ animation and support interaction, (iii) covering specific applications, and (iv) evaluation methodologies.

\section{Designing Physical Data Representations}

Three main challenges are involved in creating perceptually effective physical designs to convey digital data: $(a)$ understanding the design space of physical data representations, (b) understanding the perceptual effectiveness of different approaches, and $(c)$ finding out how to implement these designs.

\section{Charting the Design Space - Physical Variables}

Data Physicalization and Visualization share the problem of finding suitable transformations from digital data to humanreadable representations. The standard visual encoding process in Visualization maps each data point to a visual mark (i.e., a graphical primitive) and each data attribute to a visual variable (e.g., position, area, length, or hue) [13]. If we want to create physicalizations that are meant to be readable - as opposed to purely artistic data sculptures [36] - we need a similarly principled way of encoding data.

Researchers have begun to address this challenge by applying 2D and 3D visual encodings to physicalizations [69]. However, classical visual encodings largely ignore the multisensory nature of physical exploration that we previously highlighted as a benefit. Visual variables can be complemented with haptic variables such as resistance, friction, and temperature [22]. Roberts and Walker suggested the identification and use of such variables for each of our five senses [56]. However, considering senses separately does not fully capture the way information is encoded and accessed in the physical world [64]. For example, a smooth surface like glass or metal tends to be highly reflective, feels cold when touched, and silent when caressed. All these cues together participate in the perception of a single integrated physical property, "smoothness".

We argue that instead of studying individual sensory (e.g., visual or haptic) variables, it is necessary to study how they can be combined in physicalizations. We propose to call physical properties such as smoothness, hardness, or sponginess, physical variables. Physical variables are not inherently haptic, nor visual. They are also typically perceived actively, through exploratory actions involving hand, head, and body movements [20,33]. Identifying, exploring, and classifying physical variables is a research challenge that will be key to understanding the design space of data physicalizations.

\section{Understanding what is Effective}

When engaging in the design of physicalizations, one needs to know the design space of physical variables but also how effective they are at encoding and communicating data.

Perceptual studies in Visualization [13] have resulted in guidelines on how visual variables are best employed. For example, length as a visual variable is known to be perceptually effective at conveying quantitative values, while color hue is not [13]. Although initially developed for flat media, we can use many of these guidelines as a starting point for designing physicalizations. Yet, work on visualizations for wall-sized displays [6] cautions against a direct transfer. It showed that certain viewing angles change the effectiveness of visual variables and suggests that different effects may be found as well for data physicalizations. Work has also suggested that $3 \mathrm{D}$ spatial encodings are perceived more effectively when made physical than when shown on a screen [34]. 
In addition to studying how the use of visual variables transfers to non-flat spatial encodings, guidance is needed for conveying data to non-visual senses. Besides informal guidelines for haptic variables [22], we are unaware of any guideline for using physical variables. Data Physicalization needs to develop empirical guidelines similarly to those in Visualization, and consider similar questions such as: what are the just-noticeable differences that a physical variable can convey? Can it convey quantitative, ordinal, or nominal data best? Are there physical variables that can be perceived pre-attentively? Importantly, studies need to assume an active - rather than a fixed and passive - observer [20]. Also, the many interactions between the senses that physical variables address, as well as the interactions between the variables themselves, likely make their study very challenging compared to the study of visual variables.

Besides perceptual efficiency, discoverability and recognizability also need to be considered. While the presence of visual cues is easy to detect, the presence of physical cues like weight may not be. Physicalizations should be designed so that users can quickly identify which physical variables convey information, and how. This requires understanding to what extent people's experience in perceiving the physical world can be transferred to physicalizations, and how much requires explicit learning. Data physicalization researchers may need to be involved in developing new data encoding conventions, as well as helping promote physicalization literacy.

There are other aspects of data physicalization design besides the choice of appropriate physical encodings. For example physical size: a large physicalization such as in Figure 1-left can convey large amounts of data and be explored collaboratively. However, it cannot be manipulated and examined like a hand-sized physicalization [34]. Thus the effects of form factor and size will also need to be better understood.

Finally, the potential support for engagement previously discussed will also need to be studied. It is unclear which physical representations would contribute most to an engaging experience. Processing fluency - the ease with which an observer can decode an object - has also been shown to affect people's preferences [55]. Thus other qualities of physical variables need to be studied besides their perceptual effectiveness.

\section{Implementation Challenges}

Digital fabrication technologies greatly facilitated the process of prototyping and creating static physicalizations $[68,69]$ but even the most elaborate digital fabrication machines available today have not been built with data physicalization in mind. Multi-material 3D printers can interleave different source materials to gain some form of control over properties such as color, opacity, or deformability, but the range of materials available is still very limited [25,47]. Much more research is needed before machines can accurately render physical variables such as thermal conductivity, weight, smell, or taste.

A further challenge is to ensure reproduction fidelity of physical variables across different machines. Materials also need to be chosen such that the variable they encode does not "run out" or depletes due to repeated user interactions. This requires careful consideration, as certain properties like temperature or deformation can change the value of the encoded variable after successive use. One way of addressing physical variable depletion is through active physicalizations, where an external energy source can be used to maintain a physical variable such as temperature at a desired value.

Active physicalizations can address other hard problems of physicalization implementation, such as free positioning in 3D space. While an on-screen visualization can have free-floating objects, physicalizations are subject to gravity. Technologies have been explored to address this, such as the use of magnetic fields [39], bubbles [62], or acoustic levitation [61] (see Figure 1-right). However, fine control over 3D geometry is hard and no technology exists yet that can successfully physicalize data encodings as simple as 3D scatterplots.

Finally, prototyping and building physicalizations involve complex workflows, ranging from laborious and low-fidelity manual crafting to custom programming (less laborious but requires expertise) to fully automatic (lower entry requirements but little control over the design) [69]. Thus, one challenge is to characterize possible fabrication workflows and classify them as to their benefits and drawbacks, including how fast physicalizations can be created or replicated, how accurate they are, how expensive the required material is, their environmental impact, and which skills are required to create them.

\section{Supporting Animation and Interactivity}

While physical encodings are important to convey a dataset effectively, building dynamic data physicalizations can help support not only reusability across datasets, but also a wider range of analytical and communication tasks. Here, we discuss two main challenges: $(a)$ how to design effective dynamic physicalizations, and $(b)$ how to implement them.

\section{Designing Dynamic Physicalizations}

So far we considered a fixed dataset with a fixed physical representation. Most on-screen visualization systems are however dynamic, and are therefore able to visually reflect (1) changes in datasets, (2) changes in data operations such as filtering and aggregation, (3) change from one data representation to another, (4) changes in visualization settings such as color scales and sorting order, and (5) view transformations such as pan, zoom, or rotate [33]. These operations can be either triggered by the system (they are animated) or by the user (they are interactive). Much research is needed on how to support similar features on data physicalizations.

Previous work on animated visualizations examined how to turn abrupt visual changes into animated transitions [24]. Although we expect most dynamic physicalizations to support animated transitions by design, it remains a challenge to design them such that they appropriately convey the changes to the observer. In particular, it remains unknown whether optimal settings for timing apply to animations in 3D space and animations of other physical variables, and what the perceptual limits of concurrent changes in these variables are.

Designing interaction with physicalizations is likely even more challenging. As discussed before, a key characteristic of physicalizations is their built-in support for interaction techniques that generally need to be explicitly implemented on regular displays. A hand-held 3D physicalization can be manually turned 
around. Modular physicalizations can be disassembled, reassembled, rearranged, aligned, thus already covering a range of analytical tasks $[33,67]$. We call such interactions physical interactions to contrast them with what we call synthetic interactions requiring sensing, actuation, and computation.

The primary research challenge in designing effective interactive physicalizations is to find how to best combine physical with synthetic interactions. For example, with modular or malleable physicalization designs based on LEGOs or clay, a range of tasks can be manually accomplished through physical interaction, including updating datasets (see Figure 2-left), filtering data, or even changing their representation. At the same time, computer assistance is clearly desirable in many cases to accomplish repetitive or computational tasks.

Designing synthetic interaction techniques for physicalizations may require readapting - or more likely reinventing - interactive visualization. For example, should we support dynamic filtering through physical range sliders [72], or through more physical, direct manipulation gestures [18]? Some functionalities may not map to any manipulative gesture. Filtering on non-encoded dimensions, for example, will likely require alternative interaction mechanisms, such as symbolic gestures or instruments. Regardless, Data Physicalization will need to study ways of smoothly integrating synthetic with physical interaction, while avoiding "Midas Touch" issues common in gesture-based interfaces [40]. A key question is how much automation is necessary, and whether automation can be used in a way that preserves the cognitive benefits offered by physical object manipulation.

As with physical encodings, recognizability and discoverability of interactions are important. While synthetic interactions that rely on direct manipulation or familiar controls can convey their function through physical affordances (e.g., a handle or a switch), not all interaction styles can be easily expressed with affordances. Examples include symbolic and/or mid-air gestures [18]. The most effective interaction designs for data exploration also likely depend on factors such as the type of data, the size of the physicalization, and the number of users.

\section{Implementation Challenges}

While we often speak of computer displays as being dynamic displays, we want physicalizations to be more generally $r e$ configurable. Reconfigurability is required to switch datasets, to physicalize dynamic data (e.g., from a streaming source), or to get different perspectives on the same dataset. Recall that these operations can be either automatically triggered by the system (animation), or invoked by the user, either via sensing and actuation (synthetic interaction) or through purely physical manipulation (physical interaction).

Reconfigurability is required to implement rich physical interactions. For example, a modular 3D bar chart supports reordering [32], and a LEGO-based physicalization could support even more tasks. However, limited reconfigurability can be desirable. Information as to which states and operations are meaningful can be conveyed as physical constraints, by adding mechanical features such as attachments [69] or bindings [67]. Physical constraints have been already used in TUIs [72] but largely remain to be explored for physicalizations.
Actuated physicalizations can switch physical states without human intervention. Actuation provides support for animation, and once combined with sensing, for synthetic interaction. A key element to consider from the implementation perspective is the degree of self-reconfigurability of hardware technologies, as well as the speed with which the devices can reconfigure themselves. Self-reconfiguration taxonomies $[54,58]$ are key to better understanding to what extent different technologies can support data physicalization. For example, 2.5D shape displays [18] can support 3D bar charts of a certain size as well as solid terrain visualizations, but not 3D scatterplots. Although such devices are ultra-fast and can support slick interactions once combined with sensing, support for other physical representations requires a higher degree of self-reconfigurability. The technological complexity required is high, and much more research is needed to achieve full spatial flexibility [30].

Novel technologies are also needed to dynamically convey variables other than geometry. Prototypes exist that can dynamically control softness $[17,54]$ or weight [46], but more work is needed before we can "actuate" a range of physical variables such as reflectivity, porosity, rugosity or taste. The ultimate physical display that can dynamically control not only its shape and colors, but also its material properties still belongs to science-fiction, but is an exciting goal to pursue.

More work is also needed to better understand the practical trade-offs between different technologies, especially between digital fabrication and actuated devices. Despite its many benefits, actuation only supports temporal multiplexing, that is, showing a single dataset or view of a dataset over time. In contrast, many passive physicalizations (analogously to paper print-outs) can be easily duplicated, produced in different versions, arranged side-by-side, and explored. As passive physical objects they are both permanent and disposable, two properties that are lost when switching to actuated technology.

Finally, over-arching the above technological challenges is the importance of making the value of physicalizations greater than their construction cost (e.g., time, money) [74]. Only when this benefit is achieved can physicalizations become mainstream. The cost of producing data physicalizations can be reduced with appropriate tools that aid in their construction, both for static [69] and for dynamic physicalizations.

\section{Application-Specific Challenges}

Even though physicalizations promise many possible benefits, we expect them to apply differently depending on application scenarios. Thus, we need to identify the application areas that can draw immediate benefit in relation to the required cost and effort of creating them, and to understand the trade-offs involved [74]. Gaining such an understanding may also help develop physicalizations that target large audiences [14].

Consider education as an example. As mentioned earlier, physical artifacts have been shown to facilitate learning $[19,48]$. Physicalizations could further help students understand data from topics such as meteorology, history, physics, or chemistry $[15,77]$, or help them learn about data representations [75]. They could support a way of learning that is not purely auditory or visual. Students could share and compare data objects, and even author their own $[29,75]$. Yet we need 
to understand how such physicalizations should be designed to best support collaborative learning activities. It is also an open question to which extent physicalization literacy has to be taught to students before they can begin to understand physical encodings less familiar than 3D bar charts.

Using a physicalization will likely not remain a solitary activity [23]. Physical objects can easily be shared as long as collaborators are co-located [68], but in many cases people work over a distance. With current visualization tools, we can create snapshots to share by email or on paper, or to show during talks. Static physicalizations could be similarly shared through fabrication instructions, or dynamic physicalizations could be remotely synchronized. Many challenges are involved in identifying and implementing appropriate solutions.

\section{Evaluation-Specific Challenges}

Evaluating physicalizations is both important and difficult. On a methodological level, Data Physicalization shares challenges with HCI and Visualization on how to conduct evaluations.

Given the focus on data representation, many of the evaluation issues are shared by the Visualization community [37, 50,74]. A pending challenge is to find appropriate ways of studying how people engage in data exploration when no clear task is defined [10]. Another concerns the choice of criteria for assessing the merits of data representations that go beyond pure time and error metrics. For example, the benefits of the physicalizations used by Rosling (Figure 2-right) likely lie in their pedagogical and persuasive power. Other criteria relevant to physicalizations include the insights gained from interacting with them [51], the extent to which they promote engagement and behavior change, their memorability [8], and the affective responses they elicit [26]. Taking inspiration from work on visualization evaluation scenarios [37], it also seems important to explore methodologies for understanding how people reason, collaborate and communicate with physicalizations.

In terms of research methods, comparative evaluation is of importance - in particular to empirically establish the benefits of physicalizations. A difficulty lies in the proper phrasing of research questions and choice of experimental manipulations. It is important to identify a fair alternative presentation method (or modality) as a baseline of comparison. Regular computer displays are an important baseline, but others include paper and ink, whiteboards, or advanced computer displays such as stereoscopic or volumetric displays. All these baselines need to be tested before we can claim to have strong formal evidence for the merits of physicalization.

It is also crucial, yet difficult, to only manipulate the presentation modality in order to avoid experimental bias. Data encodings should be kept the same or similar across modality conditions for comparisons to be informative [34]. Support for interaction should also be kept as similar as possible. For instance, it would be unfair to compare a static physical visualization with an on-screen visualization system augmented with rich and powerful interaction techniques. Technological advances in actuated physicalizations will make it possible to support similar interaction functionality, but keeping interaction styles comparable across presentation modalities while balancing ecological validity will remain a challenge.
Another example of a potential confound is physical size. It is already known that display size can have an effect on data exploration efficiency [2]. Therefore, when evaluating the effects of physicality, physical size has to be kept the same for the comparison to be fair, e.g., wall-sized on-screen visualizations need to be compared to room-sized physicalizations.

Finally, the issues of exploring, evaluating, and implementing physicalizations all are linked and we need new methods to address them concurrently. For example, how can we investigate a range of synthetic interactions without being subject to current technical limitations? One possibility is to use proxy technologies [32], for instance, building static physicalizations for gesture elicitation studies [76] to inform the design of synthetic interactions with future actuated physicalizations.

\section{CONCLUSION}

Data Physicalization is an emerging area of research that examines how computer-supported, physical renditions of data can support cognition, communication, learning, problem solving, and decision making. It is related to and bridges traditional fields such as Visualization and Tangible User Interfaces through its combined focus on data exploration and analysis with physical data representations, we call physicalizations.

We outlined many possible benefits of physicalizations in use and multiple research challenges and opportunities that remain to be addressed. The goal of this paper is to open the discussion about Data Physicalization as a research area, by providing a first working definition of physicalization artifacts and an initial proposition of the area's research focus.

Data Physicalization is a particularly exciting research area as many questions remain unanswered and much can be learned from related disciplines. Visualization researchers, for example, can find novel contexts to apply their knowledge on the design of data representations, psychologists can contribute methodologies and studies for understanding how physical interactions influence cognition, hardware enthusiasts can work on novel ways to implement powerful shape-changing dynamic physicalizations, and Tangible and Human-Computer Interaction researchers can contribute knowledge on how to best design and implement interactions for physical data representations. There also remain many fruitful application domains to be explored for both serious data exploration with physicalizations as well as more casual use. Data Physicalization research is truly at its beginning and researchers now have the great opportunity to shape and influence it.

With this paper, we hope to inspire the creation of a community around research on Data Physicalization that will begin to address the outlined as well as new and emerging challenges and opportunities. We hope the outcome will be exciting examples of how our thinking, learning, problem solving, or communication can be enriched with data physicalizations. See the dataphys.org website for further developments.

\section{ACKNOWLEDGMENTS}

We thank Benjamin Bach for his help on the neurosurgical planning scenario. This work has been supported by the EC within the 7th framework programme through the FET Open scheme's GHOST project (grant \#309191) and by the Danish Council for Strategic Research (grant \#10-092316). 


\section{REFERENCES}

1. Alexander, J., Lucero, A., and Subramanian, S. Tilt Displays: Designing display surfaces with multi-axis tilting and actuation. In Mobile HCI (2012), 161-170.

2. Andrews, C., Endert, A., Yost, B., and North, C. Information visualization on large, high-resolution displays: Issues, challenges, and opportunities. Information Visualization 10, 4 (2011), 341-355.

3. Ascher, M., and Ascher, R. Mathematics of the Incas: Code of the Quipu. Dover Publications, 2013.

4. Barrass, S. Digital fabrication of acoustic sonifications. $J$. Audio Eng. Soc. 60, 9 (2012), 709-715.

5. Benko, H., Wilson, A. D., Zannier, F., and Benko, H. Dyadic projected spatial augmented reality. In Proc. UIST (2014), 645-655.

6. Bezerianos, A., and Isenberg, P. Perception of visual variables on tiled wall-sized displays for information visualization applications. TVCG 18, 12 (2012), 2516-2525.

7. Borghi, A. M., and Cimatti, F. Embodied cognition and beyond: acting and sensing the body. Neuropsychologia 48, 3 (2009), 763-773.

8. Borkin, M., Vo, A., Bylinskii, Z., Isola, P., Sunkavalli, S., Oliva, A., and Pfister, H. What makes a visualization memorable? TVCG 19, 12 (2013), 2306-2315.

9. Calvert, G., Spence, C., and Stein, B. E. The Handbook of Multisensory Processes. MIT Press, 2004.

10. Card, S., Mackinlay, J. D., and Shneiderman, B., Eds. Readings In Information Visualization: Using Vision To Think. Morgan Kaufmann Publishers, 1999.

11. Carello, C., Wagman, J. B., and Turvey, M. T. Acoustic specification of object properties. In Moving Image Theory: Ecological Considerations. Southern Illinois University, 2005, 79-104.

12. Challis, B. P., and Edwards, A. D. N. Design principles for tactile interaction. In Haptic Human-Computer Interaction. Springer, 2001, 17-24.

13. Cleveland, W. S., and McGill, R. Graphical perception: Theory, experimentation, and application to the development of graphical methods. J. Am. Stat. Assoc. 79, 387 (1984), 531-554.

14. Danziger, M. Information visualization for the people. Master's thesis, MIT, 2008.

15. Dragicevic, P., and Jansen, Y. List of physical visualizations. dataphys.org/list, 2012.

16. Edman, P. Tactile Graphics. AFB, 1992.

17. Follmer, S., Leithinger, D., Olwal, A., Cheng, N., and Ishii, H. Jamming user interfaces: Programmable particle stiffness and sensing for malleable and shape-changing devices. In Proc. UIST (2012), 519-528.

18. Follmer, S., Leithinger, D., Olwal, A., Hogge, A., and Ishii, H. inFORM: Dynamic physical affordances and constraints through shape and object actuation. In Proc. UIST (2013), 417-426.

19. Froebel, F. The Education of Man. A. Lovell \& Co, 1885.

20. Gibson, J. J. The Ecological Approach to Visual Perception. Psychology Press, 1986.

21. Gilpin, K., Koyanagi, K., and Rus, D. Making self-disassembling objects with multiple components in the Robot Pebbles system. In ICRA (2011), 3614-3621.
22. Griffin, A. L. Feeling it out: the use of haptic visualization for exploratory geographic analysis. Cartographic Perspectives, 39 (2001), 12-29.

23. Heer, J., Ham, F., Carpendale, S., Weaver, C., and Isenberg, P. Creation and collaboration: Engaging new audiences for information visualization. In Information Visualization, LNCS. Springer, 2008, 92-133.

24. Heer, J., and Robertson, G. G. Animated transitions in statistical data graphics. TVCG 13, 6 (2007), 1240-1247.

25. Hiller, J., and Lipson, H. Design and analysis of digital materials for physical 3d voxel printing. Rapid Prototyping Journal 15, 2 (2009), 137-149.

26. Hogan, T., and Hornecker, E. How does representation modality affect user-experience of data artifacts? In Haptic \& Audio Inter. Design. Springer, 2012, 141-151.

27. Hornecker, E. The role of physicality in tangible and embodied interactions. Interactions 18, 2 (2011), 19-23.

28. Hornecker, E., and Buur, J. Getting a grip on tangible interaction: a framework on physical space and social interaction. In Proc. CHI (2006), 437-446.

29. Huron, S., Jansen, Y., and Carpendale, S. Constructing visual representations: investigating the use of tangible tokens. TVCG 20, 12 (2014), 2102-2111.

30. Ishii, H., Lakatos, D., Bonanni, L., and Labrune, J.-B. Radical Atoms: Beyond Tangible Bits, toward transformable materials. Interactions 19, 1 (2012), 38-51.

31. Ishii, H., and Ullmer, B. Tangible Bits: towards seamless interfaces between people, bits and atoms. In Proc. CHI, ACM (1997), 234-241.

32. Jansen, Y. Physical and Tangible Information Visualization. PhD thesis, Université Paris-Sud XI, 2014.

33. Jansen, Y., and Dragicevic, P. An interaction model for visualizations beyond the desktop. TVCG 19, 12 (2013), 2396-2405.

34. Jansen, Y., Dragicevic, P., and Fekete, J.-D. Evaluating the efficiency of physical visualizations. In Proc. CHI (2013), 2593-2602.

35. Kildal, J. Kooboh: Variable tangible properties in a handheld Haptic-Illusion box. In Proc. EuroHaptics'12. Springer, 2012, 191-194.

36. Kosara, R. Visualization criticism - the missing link between information visualization and art. In Proc. IV (2007), 631-636.

37. Lam, H., Bertini, E., Isenberg, P., Plaisant, C., and Carpendale, S. Empirical studies in information visualization: Seven scenarios. TVCG 18, 9 (2012), 1520-1536.

38. Lederman, S. J., and Klatzky, R. L. Hand movements: a window into haptic object recognition. Cogn. Psychol. 19, 3 (July 1987), 342-368.

39. Lee, J., Post, R., and Ishii, H. ZeroN: Mid-air tangible interaction enabled by computer controlled magnetic levitation. In Proc. UIST (2011), 327-336.

40. Lee, J. C. In search of a natural gesture. ACM Crossroads 16, 4 (2010), 9-12.

41. McGookin, D., Robertson, E., and Brewster, S. Clutching at straws: using tangible interaction to provide non-visual access to graphs. In Proc. CHI (2010), 1715-1724. 
42. McGookin, D. K., and Brewster, S. A. SoundBar: Exploiting multiple views in multimodal graph browsing. In Proc. NordiCHI (2006), 145-154.

43. Moere, A. V. Beyond the tyranny of the pixel: Exploring the physicality of information visualization. In Proc. IV (2008), 469-474.

44. Munzner, T. Process and pitfalls in writing information visualization research papers. In Information Visualization. Springer, 2008, 134-153.

45. Murata, S., Yoshida, E., Kamimura, A., Kurokawa, H., Tomita, K., and Kokaji, S. M-TRAN: Self-reconfigurable modular robotic system. TMECH 7, 4 (2002), 431-441.

46. Niiyama, R., Yao, L., and Ishii, H. Weight and volume changing device with liquid metal transfer. In Proc. TEI (2014), 49-52.

47. Olberding, S., Wessely, M., and Steimle, J. Printscreen: fabricating highly customizable thin-film touch-displays. In Proc. UIST (2014), 281-290.

48. O'Malley, C., and Fraser, S. Literature review in learning with tangible technologies. Tech. rep., Futurelab, 2004.

49. Piper, B., Ratti, C., and Ishii, H. Illuminating clay: A 3-D tangible interface for landscape analysis. In Proc. CHI (2002), 355-362.

50. Plaisant, $\mathrm{C}$. The challenge of information visualization evaluation. In Proc. AVI (2004), 109-116.

51. Plaisant, C., Fekete, J., and Grinstein, G. Promoting insight-based evaluation of visualizations: From contest to benchmark repository. TVCG 14, 1 (2008), 120-134.

52. Poupyrev, I., Nashida, T., Maruyama, S., Rekimoto, J., and Yamaji, Y. Lumen: interactive visual and shape display for calm computing. In SIGGRAPH Emerging Technologies (2004), 17.

53. Pousman, Z., Stasko, J. T., and Mateas, M. Casual information visualization: Depictions of data in everyday life. TVCG 13, 6 (2007).

54. Rasmussen, M. K., Pedersen, E. W., Petersen, M. G., and Hornbæk, K. Shape-changing interfaces: a review of the design space and open research questions. In Proc. CHI (2012), 735-744.

55. Reber, R., Schwarz, N., and Winkielman, P. Processing fluency and aesthetic pleasure: Is beauty in the perceiver's processing experience? Personality and Social Psychology Review 8, 4 (2004), 364-382.

56. Roberts, J. C., and Walker, R. Using all our senses: the need for a unified theoretical approach to multi-sensory information visualization. In VisWeek Workshop (2010).

57. Romanishin, J., Gilpin, K., and Rus, D. M-blocks: Momentum-driven, magnetic modular robots. In Proc. IROS, IEEE/RSJ (2013), 4288-4295.

58. Roudaut, A., Karnik, A., Löchtefeld, M., and Subramanian, S. Morphees: Toward high "shape resolution" in self-actuated flexible mobile devices. In Proc. CHI (2013), 593-602.

59. Schmandt-Besserat, D. How Writing Came About. University of Texas Press, 1996.

60. Schweikardt, E., and Gross, M. D. roBlocks: A robotic construction kit for mathematics and science education. In Proc. ICMI (2006), 72-75.

61. Seah, S., Drinkwater, B., Carter, T., Malkin, R., and Subramanian, S. Dexterous ultrasonic levitation of millimeter-sized objects in air. UFFC 61, 7 (2014), 1233-1236.
62. Seah, S. A., Martinez Plasencia, D., Bennett, P. D., Karnik, A., Otrocol, V. S., Knibbe, J., Cockburn, A., and Subramanian, S. SensaBubble: A chrono-sensory mid-air display of sight and smell. In Proc. CHI (2014), 2863-2872.

63. Shaer, O., and Hornecker, E. Tangible user interfaces: Past, present, and future directions. Found. Trends Hum. -Comput. Interact. 3 (2010), 1-137.

64. Spelke, E. S. The development of intermodal perception. In Handbook of Infant Perception. Academic Press, 1987, 233-273.

65. Steimle, J., Jordt, A., and Maes, P. Flexpad: highly flexible bending interactions for projected handheld displays. In Proc. CHI (2013), 237-246.

66. Stusak, S., and Aslan, A. Beyond physical bar charts: An exploration of designing physical visualizations. In Proc. CHI-EA (2014), 1381-1386.

67. Stusak, S., Tabard, A., and Butz, A. Can physical visualizations support analytical tasks? In Posters of IEEE InfoVis (2013).

68. Stusak, S., Tabard, A., Sauka, F., Khot, R., and Butz, A. Activity sculptures: Exploring the impact of physical visualizations on running activity. TVCG 20, 12 (2014), 2201-2210.

69. Swaminathan, S., Shi, C., Jansen, Y., Dragicevic, P., Oehlberg, L., and Fekete, J.-D. Supporting the design and fabrication of physical visualizations. In Proc. $\mathrm{CHI}$ (2014), 3845-3854.

70. Tang, S. K., Sekikawa, Y., Leithinger, D., Follmer, S., and Ishii, H. Tangible cityscape, 2013. tinyurl.com/tcityscape.

71. Ullmer, B., and Ishii, H. Emerging frameworks for tangible user interfaces. IBM Syst. J. 39 (July 2000).

72. Ullmer, B., Ishii, H., and Jacob, R. J. K. Tangible query interfaces: Physically constrained tokens for manipulating database queries. In Proc. INTERACT (2003), 279-286.

73. Underkoffler, J., and Ishii, H. Urp: A luminous-tangible workbench for urban planning and design. In Proc. CHI (1999), 386-393.

74. Van Wijk, J. J. The value of visualization. In Proc. VIS (2005), 79-86.

75. Vande Moere, A., and Patel, S. The Physical Visualization of Information: Designing Data Sculptures in an Educational Context. In Visual Information Communication. Springer, 2010, pp. 1-23.

76. Wobbrock, J. O., Morris, M. R., and Wilson, A. D. User-defined gestures for surface computing. In Proc. CHI (2009), 1083-1092.

77. Wu, H.-K., and Shah, P. Exploring visuospatial thinking in chemistry learning. Science Education 88, 3 (2004), 465-492.

78. Yao, L., Niiyama, R., Ou, J., Follmer, S., Della Silva, C., and Ishii, H. PneUI: Pneumatically actuated soft composite materials for shape changing interfaces. In Proc. UIST (2013), 13-22.

79. Zhao, J., and Vande Moere, A. Embodiment in data sculpture: A model of the physical visualization of information. In Proc. DIMEA (2008), 343-350. 